\title{
Les Centres Éducatifs Familiaux de Formation par Alternance en Afrique: le cas du Cameroun
}

\author{
Benoît Birwe ${ }^{1}$, Pedro Puig-Calvó ${ }^{2}$ \\ ${ }^{1}$ Plateforme pour la Promotion des Centres Éducatifs Familiaux de Formation par Alternance au Cameroun - PROCEFFA. \\ Yaounde (Biyem-Assi Maison Blanche). Cameroon, Africa. ${ }^{2}$ Universitat Internacional de Catalunya - UIC / Association \\ Internationale des Mouvements Familiaux de Formation Rurale - AIMFR, Spain. \\ Auteur correspondant/Author for correspondence: birwebenoit@yahoo.fr
}

RÉSUMÉ. Cet article questionne la durabilité des dispositifs de formation professionnelle par alternance que sont les Centres Éducatifs Familiaux de Formation par Alternance (CEFFA) en Afrique en général et, de manière particulière au Cameroun, où ils sont confrontés aux difficultés réelles pour assurer leur autonomisation. Les CEFFA, dont la formule d'éducation va se répandre à travers le monde tel un essaim d'abeilles grâce à la coopération entre la France et des pays partenaires, peinent encore à s'enraciner ou à s'ancrer dans le territoire. L'analyse est davantage focalisée sur la viabilité financière, en tant qu'aptitude des associations promotrices de CEFFA à assurer leur autonomie de fonctionnement en disposant des ressources nécessaires pour couvrir toutes les charges. L'article est à la fois un travail de recherche historique et d'analyse des principales difficultés d'ancrage territorial des CEFFA dans le contexte africain et des défis liés à une implantation durable des CEFFA au Cameroun.

Mots-clés: Cameroun, Formation Professionnelle, Formation Intégrale, Alternance, Associations de Familles, Organisation Promotrice, Durabilité. 


\title{
The Family Farm Schools in Africa: the case of Cameroon
}

\begin{abstract}
This article questions the sustainability of dual vocational training schemes that are Family Farm Schools (FFS) in Africa in general and, in a particular way in Cameroon, where they face real difficulties to ensure their empowerment. These Family Farm Schools, whose educational formula will be spread throughout the world like a swarm of bees thanks to the cooperation between France and partner countries, still struggle to take root sustainably. The analysis focuses more on financial sustainability, as the ability of Family Farm School's Promoter Organizations to ensure their operational autonomy by having the necessary resources to cover all expenses. The article aims to be both a historical research and analysis of the main difficulties anchoring the Family Farm Schools in the African context and challenges to a sustainable implementation of the Family Farm Schools in Cameroon.
\end{abstract}

Keywords: Cameroon, Vocational Training, Integral Formation, Alternance, Parent Associations, Promoter Organizations, Sustainability. 


\section{Las Escuelas Familiares Agrícolas en África: el caso de Camerún}

RESUMEN. Este artículo cuestiona la sostenibilidad de los dispositivos de formación profesional por alternancia que son los Centros Educativos Familiares de Formación por Alternancia (CEFFA) en África en general y, en Camerún en particular, donde se ven confrontados a las dificultades reales para asegurar su autonomización. Estos CEFFA, la fórmula educativa de los cuales se expandió por todo el mundo como un enjambre de abejas gracias a la cooperación entre Francia y países colaboradores, a duras penas han conseguido enraizarse $\mathrm{o}$ anclarse en el territorio. El análisis se focaliza sobretodo en la viabilidad financiera, en tanto que aptitud de las asociaciones promotoras de CEFFA el asegurar su autonomía de funcionamiento disponiendo de los recursos necesarios para cubrir todas las cargas. El artículo es, al mismo tiempo, un trabajo de investigación histórica y de análisis de las principales dificultades de anclaje territorial de los CEFFA en el contexto africano y de los desafíos relativos a una implantación duradera de los CEFFA en Camerún.

Palabras clave: Camerún, Formación Profesional, Formación Integral, Alternancia, Asociación de Familias, Organización promotora, Sostenibilidad. 


\section{Présentation}

L'histoire des premières Maisons Familiales Rurales en France et dans le monde est un exemple de dignification des personnes du milieu rural à travers la formation (Granereau, 2007). C'est en Tunisie, puis en Algérie que sont créés les premiers CEFFA en Afrique (NovéJosserand, 2009). Des responsables français du Mouvement des MFR étaient envoyés en Afrique avec pour mission de former les formateurs et les associations de familles. Puis, des partenariats, inscrits au registre des actions de coopération cofinancées par l'Agence Française de Développement (AFD), les fédérations des MFR de France ou la Fondation des MFR dans le Monde étaient initiés entre les MFR françaises et celles d'Afrique dans l'optique d'apporter une assistance technique et pédagogique, de consolider et de développer l'action des MFR à l'international en faveur de la formation des jeunes en milieu rural. Au Cameroun, les CEFFA $^{\mathrm{i}}$, connus sous diverses appellations (EFA, EFR, MFR, IFER, FFS ...), sont des centres de formation générale et professionnelle par alternance en étroite relation avec les réalités du milieu, promus par des organisations de la société civile (organisations confessionnelles, réseaux d'écoles, ONG, organisations de producteurs, société...) ou des associations de familles. Ces centres offrent aux jeunes sans qualification professionnelle ${ }^{\mathrm{ii}}$ des milieux ruraux et périurbains - le plus souvent déscolarisés - et aux producteurs en activitéiii, une gamme variée de formation: agriculture-élevage, hôtellerierestauration, esthétique-coiffure, industrie d'habillement, métier de bois, électricité, secrétariat bureautique.

En outre, pour valoriser la polyvalence et la pluriactivité, caractéristique des zones rurales, d'autres savoir-faire y sont enseignés, notamment l'apiculture, la transformation agroalimentaire, les apprentissages pratiques divers...pour permettre aux jeunes de diversifier leurs revenus. Les CEFFA sont ainsi une opportunité offerte aux jeunes de se former dans un métier et d'être accompagnés à l'insertion socioprofessionnelle sur leur territoire en emploi salarié, auto-emploi ou poursuite des études.

$\mathrm{Si}$ les CEFFA contribuent à la formation et à l'insertion professionnelle des jeunes en milieu rural au Cameroun depuis 27 ans déjà, il se pose encore avec acuité le problème de la durabilité de ces dispositifs de formation et d'insertion des jeunes, calqués sur le modèle originel français. Comment assurer la durabilité de ces dispositifs de formation professionnelle par alternance? Quelle stratégie adopter? 
A quelles conditions les CEFFA peuventils devenir durables ? Quand et comment furent implantés les CEFFA au Cameroun? Comment les familles s'approprient-elles de ces dispositifs de formation et d'insertion professionnelle des jeunes ? Pour répondre à ces questions, une première partie retracera brièvement l'historique des CEFFA au Cameroun. La seconde partie présentera la philosophie de l'éducation des CEFFA; tandis que la troisième partie sera consacrée aux défis. La dernière partie expose quelques initiatives prises en matière d'autonomisation des CEFFA au Cameroun.

\section{Bref historique des CEFFA au Cameroun}

L'histoire des CEFFA au Cameroun peut être reconstituée en deux temps: d'abord, une première tentative manquée; ensuite, une nouvelle page de l'histoire.

\section{Une tentative manquée}

C'est d'abord un député, originaire d'Abong-Mbang qui, ayant visité les Maisons Familiales Rurales (MFR) françaises, crée en 1964 un CEFFA qui fermera ses portes en 1966, faute d'implication des familles. Ensuite, c'est au tour du feu Mgr Jean Zoa, qui lui aussi a visité les MFR en France, de mener la campagne de sensibilisation sur les MFR dans les diocèses et paroisses. En 1968 à Abong-Mbang et en 1969 à Diang, furent créés des Centres d'éducation et de formation professionnelle agricole à la demande adressée à l'UNMFREO par un paysan camerounais actif au sein du Mouvement International de la Jeunesse Agricole et Rurale Chrétienne (MIJARC) qui a connu les MFR lors d'une mission de découverte en France (Le Bissonnais \& Erceau, 2014). Cette première tentative s'est malheureusement soldée par un échec. L'année 1974 marque définitivement la fin de cette expérience, faute de soutien financier (l'engagement des MFR françaises s'étant limité à six ans) et du manque d'intérêt des pouvoirs publics camerounais.

\section{Une nouvelle page de l'histoire}

À partir de 1992, l'Institut Européen de Coopération et de Développement (IECD) prend la relève dans la création des Écoles Familiales Agricoles (EFA). Cette phase, dont l'objectif était le développement et l'essaimage des EFA, a connu la création, entre autres, de l'EFA d'Esse (1992), l'EFA d'Eyen-Meyong (1996), l'EFA d'Apouh (1998). Ces EFA vont ensuite être regroupées au sein d'une Union Nationale des Écoles Familiales Agricoles du Cameroun (UNEFAC). 
Sous l'impulsion de l'IECD, une organisation d'assistance technique à un réseau d'EFA dénommée Coordination Nationale des Écoles Familiales Agricoles $\mathrm{du}$ Cameroun (CNEFAC) est officiellement créée le 24 août 2004. Quelques années plus tard, le 20 novembre 2008 est portée des fonds baptismaux l'Association des Centres Familiaux de Formation par Alternance (ACEFFA) ${ }^{\text {iv }}$ qui ne fonctionnera que jusqu'en 2010. Cette association bénéficiait de l'appui technique de la CNEFAC. C'est alors que les EFA seront regroupées en fédérations régionales, membres de la CNEFAC.

En 1998, l'UNMFREO est à nouveau sollicitée au Cameroun, avec l'appui de l'association Agriculteurs Français et Développement International (AFDI). Le partenariat entre le Cameroun et la Fédération Régionale des MFR d'Auvergne aboutit à la création en 2005 de la Fédération des Écoles et Maisons Familiales Rurales du Cameroun (FEMAFARC). Cette dernière promeut des Maisons Familiales Rurales et des Écoles Familiales Rurales : MFR de Ndognihbe (2005) transférée à Massok dont le nom deviendra MAFARSAM, EFR de Bafia (2005), EFR de Yenga, EFR de Mang, EFR de Kaélé.
En 2014, l'ONG belge DISOP, fort de son expérience du Mouvement des CEFFA en Amérique latine (notamment au Brésil) et qui soutenait dès 2008 le projet de promotion des Écoles Familiales Agricoles à 1'Est du Cameroun ${ }^{v}$, initie un Programme triennal de promotion des CEFFA, dénommé DEFIS-Jeunes Cameroun, dont la première phase (20142016) fut celle de l'expérimentation et de la validation de la pédagogie de l'Alternance, l'asbl DISOP va impulser et soutenir la mise en place de la Plateforme pour la Promotion des Centres Éducatifs Familiaux de Formation par Alternance (PROCEFFA) au Cameroun.

Depuis lors, les CEFFA se structurent. Les organisations promotrices de CEFFA au Cameroun, dont la plupart sont partenaires de l'ONG belge VIA Don Bosco dans le cadre du Programme 20172021, sont regroupées au sein d'une plateforme des acteurs de la formation par alternance (PROCEFFA) afin de mutualiser leurs expériences, de mettre en synergie leurs compétences et de mener le plaidoyer auprès des pouvoirs publics pour la reconnaissance des CEFFA et de la formation par alternance. Cependant, malgré la bonne intention des coopérants français de l'époque de «vulgariser» les CEFFA et l'engouement des populations, il n'était pas exclu que certains acteurs 
(autorités locales, promoteurs, bénéficiaires) étaient davantage attirés par les financements qu'ils pouvaient capter grâce aux MFR que par le projet éducatif en lui-même. Ce qui plombait le projet quelques années seulement après sa mise en place. Le Projet qui désigne un but que l'on se propose d'atteindre ou bien une idée de quelque chose à faire, dans le monde des projets de coopération est plutôt interprété comme une source de ressources financiers.

\section{La philosophie de l'éducation des CEFFA}

L'alternance des CEFFA est à la fois une pédagogie et une philosophie de l'éducation en ce sens que la formation des jeunes, organisée selon le principe de l'alternance, est sous-tendue par une certaine conception de l'homme.

\section{Une pédagogie ou un système éducatif?}

Selon Jean-Claude Gimonet (Gimonet, 2010), la formation par alternance bénéficie de nos jours d'un regain d'intérêt dans les systèmes éducatifs européens, notamment français et, partant en Afrique francophone. L'on s'interroge, formule des hypothèses, construit « des ingénieries pédagogiques » et théorise au cours des colloques, séminaires, groupes de travail, dans les articles, les revues et les livres car, l'alternance en formation est loin d'avoir livré tous ses secrets. Elle est toujours en construction et en permanente adéquation avec la réalité.

L'alternance n'est pas une simple méthode ou procédure pour répondre aux difficultés du système éducatif et au problème d'insertion professionnelle des jeunes. Ce serait une vision trop simpliste, voire réductrice de l'alternance. Elle est un système pédagogique complexe qui va audelà du triangle pédagogique classique (Professeur-Élève-Savoir) au sein d'une classe. La dynamique de la pédagogie active, elle aussi, ne suffit pas; il faut entrer dans la pédagogie expérientielle, la pédagogie de la complexité. Avec l'Alternance, on est embarqué dans l'aventure de l'éducation systémique (Rosnay, 1975).

La réalité est si complexe que si on veut saisir les procédés de la formation alternée, il convient d'en définir les composantes et leurs interactions, de les hiérarchiser et de les organiser dans une perspective systémique et complexe, on peut aussi la classer en droite ligne de Morin l'alternance comme une pédagogie de la complexité. (Morin, 1990). C'est «alors seulement, on délaisse la simple méthode pédagogique pour envisager la mise en ouvre d'un système éducatif» (Gimonet, 2010). On surpasse les 
alternances juxtapositives ou administratives (Bourgeon, 1979) pour rechercher une alternance intégrative, c'est-à-dire la vraie alternance selon l'expression de Daniel Chartier (Chartier, 1986) ou plutôt l'alternance interactive (Puig-Calvó, 2006), dans laquelle tous les acteurs et éléments interagissent entre eux.

\section{Une approche intégrale et humaniste de la formation}

La formation dispensée dans les CEFFA vise l'intégralité de la personne qu'il s'agit d'appréhender dans toutes ses dimensions : intellectuelle, professionnelle, sociale, humaine, éthique, spirituelle ... L'option pour une formation associée où la formation professionnelle est au service de la formation générale du jeune y contribue grandement. Il s'agit certes de former un professionnel du métier, mais davantage un homme qui sera ensuite un professionnel. De même, l'on ne doit pas méconnaître l'importance de la vie à l'internat comme lieu d'apprentissage de la vie sociale, de la citoyenneté, de l'ouverture socioculturelle, de la compréhension et du respect de l'autre.

Dans les CEFFA l'éducation et la formation des jeunes et adultes, doit mettre au centre la personne dans son intégralité, avec une vision transcendantale de la personne et d'une façon transversale la responsabilité écologique, pour soutenir la terre et l'univers. (François, 2015), nécessaire la sauvegarde de la maison commune.

Figure 1 - Formation Integrale.

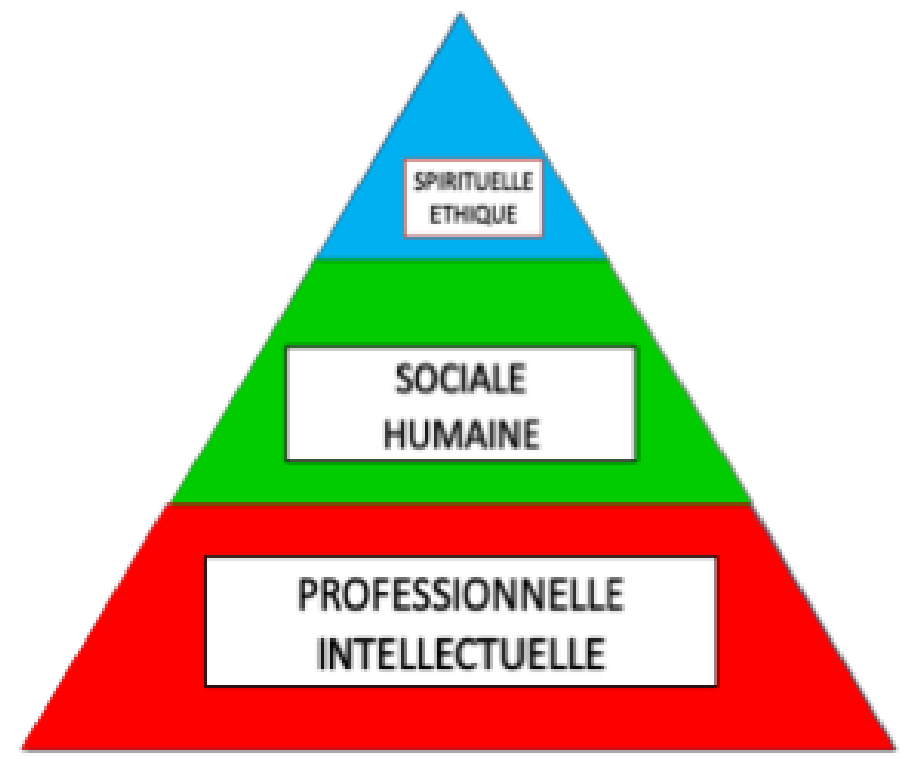

Source: Pedro Puig-Calvó (Puig-Calvó, 2019). 
Un ancrage personnaliste de la formation

L'approche éducative dans les CEFFA est fortement empreinte du courant personnaliste d'Emmanuel Mounier ${ }^{\text {vi }}$ qui a exercé une grande influence sur la pensée d'un des pionniers de la pédagogie de l'alternance des CEFFA, à savoir André Duffaure $^{\text {vii }}$. En fait, selon le philosophe français Emmanuel Mounier «l'homme est-être-dans-le-monde. Sa condition ne peut être saisie, sans être aussitôt saisie comme condition incarnée et insérée. Pas plus qu'elle n'existe et ne vit indépendamment des autres, la personne ne vit et n'existe indépendamment de la nature. Aussi doit elle se réaliser par le corps-à-corps, aussi bien que par la vie intérieure » (Mounier, 2001) Cette conception se trouve être au fondement même de la pédagogie de l'alternance des CEFFA qui centre son action pédagogique sur la relation de l'apprenant avec son propre milieu (social et professionnel). L'action pédagogique de l'alternance valorise ainsi cette relation et en fait le cœur de toute l'organisation pédagogique.

En outre, le personnalisme de Mounier, distinct de toute forme d'individualisme viii, est à l'antipode de l'emprise excessive de la technologie pour valoriser la créativité, la liberté et la singularité de la personne. Ce qui transparaît clairement dans le type d'accompagnement individualisé à travers le tutorat que les moniteurs assurent aux apprenants dans les CEFFA. Le jeune en formation est appelé, non seulement à être acteur de sa formation et créateur de sens, mais également à découvrir ses talents et atouts, d'exprimer sa créativité, de développer ce qu'il a de singulier, de révéler ses potentialités, d'être considéré comme une personne unique et de l'accompagner à son rythme.

$\mathrm{Au}$ CEFFA, des activités telle la mise en commun des études d'alternance, de retour du séjour en stage professionnel, permet à l'apprenant d'extérioriser/verbaliser son vécu et d'être constructeur de sens. De même, la formation dispensée dans les CEFFA ne se limite pas à l'enseignement pour embrasser l'éducation de tout l'homme.

Les CEFFA doivent aussi certains de leurs principes éducatifs au Mouvement de la Jeunesse Agricole Chrétienne théorisée à travers la méthode du Voir-Juger-Agir où voir voudrait dire l'observation attentive de la réalité par l'apprenant qui est face au réel le plus concret (Ichard, 2015); une réalité professionnelle, sociale et familiale qu'il doit par lui-même découvrir. Juger, c'est être capable de se poser des questions à partir des faits observés, être amené à proposer une solution. Enfin, agir ouvre sur les actions concrètes en vue de la 
transformation sociale, de la prise d'initiatives et des responsabilités (Sanselme, 2000) il y a donc une synergie entre les idées du catholicisme social de cette époque, dans sa variante personnaliste, et le projet éducatif des CEFFA desquelles ils sont issus en 193537.

Par ailleurs, les doctrines pédagogiques de l'éducation nouvelle, notamment certains auteurs comme Roger Cousinet $^{\mathrm{ix}}$ avec entre autres éléments la méthode de travail par groupes, préfigurateur de la pédagogie du projet (Cousinet, 2011), John Dewey ${ }^{\mathrm{x}}$, spécialiste en psychologie appliquée et en pédagogie que dans son livre «Démocratie et Éducation » propose de centrer l'éducation sur les activités sociales plutôt que sur les savoirs ou l'importance de rétablir la continuité de l'école et de la société (Dewey, 2018), De même Carl Rogers ${ }^{x i}$ nous signale que l'enseignant ne doit pas être "un maître à penser" mais plutôt un "facilitateur d'apprentissage". (Rogers, 2013) Tous ont nourri le projet éducatif des CEFFA, D'autres auteurs plus récents comme le Brésilien, Paulo Freire (Freire, 2019) un des plus grands éducateurs universels, avec son ouvrage majeur «La pédagogie des opprimés», expose le concept appelé en portugais “conscientização" qu'on peut traduire par "conscience critique", a contribué, et encore permettent d'avancer et la construction permanente du système de formation et développement qu'est l'alternance des CEFFA.

\section{Quelques défis des CEFFA au Cameroun}

$\mathrm{Si}$ la qualification professionnelle s'acquiert autant par la formation que par l'expérience professionnelle, les CEFFA en alternant le temps de formation entre le milieu socio-professionnel et le centre de formation professionnelle peuvent se targuer de disposer d'une méthodologie efficace pour qualifier les jeunes et remédier efficacement au problème de l'inadéquation formation-emploi. De nombreux défis se pointent ainsi à l'horizon.

\section{La formation des formateurs}

Les formateurs, appelés moniteurs, constituent la cheville ouvrière du système d'alternance. Mais, pour pouvoir jouer ce rôle qui est le leur, la formation des formateurs est une condition sine qua non. Elle vise à ce que les moniteurs maîtrisent les outils de l'alternance et appliquent la démarche pédagogique d'une alternance intégrative mais, viser un modèle d'alternance intégrative (Vanhulle, Merhan, \& Ronveaux, 2007) (Malglaive, 
1994) implique dépasser la dichotomie entre théorie et pratique et de remettre en question les hiérarchies, entre les deux lieux de formation.

Certes, des sessions de formation pédagogique et technique sont régulièrement organisées, autant par les réseaux que par la plateforme nationale, à l'endroit de ces moniteurs, mais la certification de ces parcours de formation demeure encore une autre paire de manches. Par ailleurs la profession d'enseignant n'étant pas assez valorisée au niveau national, les formateurs sont toujours financés par les «projets de coopération » Mais les résultats, loin d'être une évidence -étant donné les multiples difficultés-, nous montrent qu'on a besoin d'approfondir la nature de la relation dans la formation par alternance cette relation « interactive » (Puig-Calvó, 2006) entre la théorie et la pratique, spécialement dans le contexte éducatif, social et politique africain.

\section{La motivation des maîtres de stage en question}

$\mathrm{Au}$ Cameroun comme partout ailleurs dans les CEFFA, les stages professionnels se font généralement en familles ou dans une exploitation agricole, un atelier de couture, un salon d'esthétique-coiffure, un établissement hôtelier. En général, les apprenants y séjournent deux semaines pour acquérir le geste professionnel auprès d'un maître d'apprentissage qu'est une personne qui vit de son métier. Alors que les apprenants apprécient fortement les séjours en milieu socio-professionnel où ils apprennent les ficelles du métier, ils trouvent, par contre, les séjours en familles peu formatifs, surtout lorsque la famille dont est issu le jeune en formation ne fait pas dans la culture/élevage ou simplement le métier pour lequel est envoyé en stage professionnel.

Par ailleurs, face aux initiatives concurrentes, notamment les structures étatiques (Fonds National de l'Emploi) qui financent les stages en entreprise des jeunes qu'ils accueillent, les maîtres de stage, en milieu urbain particulièrement, ont de plus en plus tendance à exiger une rémunération des CEFFA, par comparaison à la contrepartie qui leur est versée par les structures gouvernementales ou en arguant que tout apprenant qu'il forme est leur potentiel concurrent. Cela constitue un véritable problème pour les CEFFA à l'heure où les financements se raréfient et en dépit de la contrepartie en travail effectué par les apprenants en stage.

Le rôle des associations de familles dans la gouvernance des CEFFA 
Les CEFFA sont, par essence, des centres de formation par alternance créés par les familles constituées en association. Dans le modèle originel français, l'association de familles est au cœur du projet de création de tout CEFFA en ce sens que c'est elle qui crée, gère et approuve le plan de formation.

Certes, le Cameroun ne déroge pas à cette règle, mais à côté des CEFFA créés par les associations de familles, des initiatives individuelles (promoteurs), des entreprises $\left(\right.$ SAFACAM, PHP) ${ }^{\mathrm{xii}}$ ou des organisations de la société civile ont pris corps, avec l'appui des partenaires techniques et financiers. Dans ces cas d'espèce, le positionnement, quelquefois incertain, de l'association de familles dans le fonctionnement du CEFFA explique le déficit d'appropriation de ces initiatives par les communautés des localités d'implantation de ces CEFFA. Ce qui fera dire à Blaise Ango, en partant du contexte camerounais, que le portage associatif des CEFFA demeure fragile et inefficace (Ango, 2017). Un travail de fond s'avère alors nécessaire à effectuer avec ces promoteurs. Pourtant, l'implication des familles est un vecteur indispensable pour l'insertion socio-professionnelle des jeunes formés en termes d'accès au foncier, d'aide à l'installation, d'appui matériel ou de soutien moral dans la mise en œuvre des projets des jeunes.

\section{L'autonomisation des CEFFA}

La plupart des CEFFA au Cameroun fonctionnent grâce à l'appui technique et financier des organisations étrangères. Ils dépendent ainsi largement des financements extérieurs. Cette forte dépendance des ressources extérieures ou cette absence généralisée d'autonomie financière remet en cause la pérennité de ces CEFFA qui ne dépassent pas la durée de vie des financements extérieurs. On comprend alors pourquoi des CEFFA clôturent leurs activités de formation aussitôt que l'appui financier extérieur tarit.

Face à cette situation, il s'est avéré impérieux pour les organisations promotrices de CEFFA de se doter d'un plan d'autonomisation de leurs CEFFA. Ces initiatives d'autonomisation prennent quelquefois la forme d'une écoleentreprise avec une visée à la foi économique et pédagogique.

\section{L'accompagnement post-formation des jeunes: insertion socio-professionnelle}

Se limiter à assurer aux jeunes des CEFFA une formation professionnelle et technique, fût-elle de qualité, sans leur ouvrir des perspectives d'intégration en 
emploi s'est révélé inefficace dans le contexte camerounais où le chômage est grandissant et les métiers agricoles peu attractifs pour les jeunes en milieu rural. Désormais, les CEFFA doivent étendre l'accompagnement des jeunes à la phase post-formation afin de les aider à trouver des mécanismes de financement (accès à des subventions ou crédits ...) ou des appuis multiformes pour leurs projets professionnels. C'est dans le même sillage que les organisations promotrices, sur la base des expériences des organisations partenaires de VIA Don Bosco au Bénin et au Mali, ont amorcé depuis peu la réflexion sur la mise en place des Bureaux d'Emploi (BdE) dans le but de favoriser l'accès à l'emploi pour les jeunes, de les accompagner à l'insertion socioprofessionnelle en les mettant en relation avec les entreprises offrant des opportunités d'emploi.

Vers une pérennisation des CEFFA: des actions initiées

La diversification des ressources, un gage de la pérennité

Les organisations promotrices de CEFFA au Cameroun sont de plus en plus conscientes de l'intérêt de diversifier leurs partenaires techniques et financiers afin de garantir la poursuite des activités de formation quand bien même les financements extérieurs seront ou sont suspendus. Certaines d'entre elles, avec l'accompagnement de la PROCEFFA ou non, sont déjà engagées sur cette voie du renforcement des dispositifs existants. En témoigne la signature des récentes conventions entre la CNEFACMINADER, FEMAFARC-MINADER ou CNEFAC-MINJEC qui, cependant tardent encore à tenir les fleurs de la promesse.

\section{Le travail de plaidoyer auprès des pouvoirs publics pour la reconnaissance des CEFFA}

La durabilité des CEFFA dépend aussi pour une bonne part de la capacité des centres à délivrer des certificats et diplômes reconnus par l'État aux jeunes qui y sont admis. La mise en place d'une plateforme nationale dénote certainement de cette volonté des Organisations Promotrices de CEFFA au Cameroun non seulement d'échanger les expériences et de capitaliser les bonnes pratiques, mais surtout de peser auprès des pouvoirs publics afin d'obtenir une reconnaissance. Ce qui leur permettrait en outre d'obtenir des financements nationaux et jouer un rôle stratégique dans les politiques de formation agricole et rurale.

La recherche de l'articulation avec les politiques publiques de formation et d'insertion professionnelle 
Certaines organisations ont opté pour l'intégration des CEFFA qu'elles promeuvent dans les dispositifs publics existants d'appui à la formation professionnelle. Ainsi a-t-on vu des CEFFA intégrés dans le portefeuille du Programme AFOP qui réunit une centaine de centres de formation agricole au Cameroun. Cela constitue certes une solution, mais qui n'est pas toujours durable ni sans risque lorsque l'on clame son appartenance au Mouvement International des CEFFA qui a ses principes éducatifs et pédagogiques. Donc les finalités de la formation intégrale du jeune et du développement des territoires avec la participation des familles et un système pédagogique propre ne sont pas toujours bien acceptées.

\section{Conclusion}

En somme, le projet éducatif des CEFFA est un pari. Il traduit un engagement des familles, des maîtres de stage et des professionnels du milieu pour accompagner chaque jeune vers l'édification de son avenir ; un engagement sous-tendu par une vision d'une formation intégrale de l'homme qu'il s'agit de développer dans ses dimensions humaine, technique, professionnelle, sociale, intellectuelle ou spirituelle. Cet engagement collectif, associatif ne se concrétisera alors que si les associations promotrices de CEFFA, les familles et autres acteurs du milieu œuvrent ensemble pour l'épanouissement du jeune dont l'avenir est assuré. En outre, pour que ce système éducatif des CEFFA soit durable en contexte africain, de nombreux défis sont à relever ensemble, notamment l'enracinement du modèle dans son contexte, la reconnaissance de la formation que délivrent les CEFFA, l'implication des familles dans l'éducation de leurs jeunes, le soutien des pouvoirs publics à ces dispositifs de formation professionnelle des jeunes en milieu rural que sont les CEFFA. C'est à ces conditions que les CEFFA participeront au développement des territoires où ils sont implantés. En dépit de ces nombreux défis pour assurer la durabilité des CEFFA au Cameroun, l'existence d'une plateforme nationale qui accompagne les organisations promotrices de CEFFA à l'élaboration des stratégies d'autonomisation, la reconnaissance de l'alternance dans le texte régissant la formation professionnelle au Cameroun sont des avancées majeures et demeurent des leviers sur lesquels il faut actionner. Par ailleurs, il convient de noter que la formation professionnelle, autant que l'insertion professionnelle des jeunes (en emploi ou en auto-emploi) est un processus qui s’inscrit dans la durée. Ce qui soulève 
alors la question des limites des financements de courte durée qui ne permettent pas de créer des conditions favorables à la pérennisation du dispositif.

\section{Références}

Ango, B. (2017). S'adapter aux nouveaux acteurs agricoles et ruraux. Paris: UNMFREO.

Bourgeon, G. (1979). Sociopèdagogie de l'Alternance. Paris: Messonance.

Chartier, D. (1986). A l'aube des formations en alternance. París: editions Universitaires.

Cousinet, R. (2011). Une méthode de travail livre par groupes. Paris: Editions Fabert.

Dewey, J. (2018). Démocratie et éducation. Suivi de Expérience et éducation. Paris: Armand Colin.

François, P. (2015). Laudato si. Roma: Editrice Vaticana.

Freire, P. (2019). La Pédagogie des Opprimés. Récupéré sur https://resistance71.wordpress.com/paulofreire-la-pedagogie-des-opprimes-denouveau-en-francais-pdf-gratuit/: https://resistance71.wordpress.com/paulofreire-la-pedagogie-des-opprimes-denouveau-en-francais-pdf-gratuit/

Geay, A. (1998). L'école de l'alternance. Paris: l'Harmattan.

Gimonet, J. C. (2010). L'Alternance en Formation: Methode Pedagogique ou nouveau Systeme Educatif. Paris: AIMFR.

Granereau, A. (2007). Le livre de Lauzun. Paris: L'Harmattan.
Ichard, J. L. (2015, décembre). La Formation: un facteur clé $d u$ développenment rural. Récupéré sur https://www.tdx.cat/bitstream/handle/1080 3/384714/Tesi_Jean_Louis_Ichard.pdf?seq uence=9:

Le Bissonnais, A., \& Erceau, J. (2014). Les Masions Familiales Rurales dans le monde: une contributionoriginalesà la formation et à l'insertion des jeunes. Paris: L'Harmattan.

Lesourd, F. (2009). L'homme en transition. Éducationet tournants de vie. Paris: Anthropos.

Malglaive, G. (1994). Alternance et Compétences. Les Cahiers Pédagogiques (320), 26-28.

Morin, E. (1990). Introduction à la pensée complexe. Paris: du Seuil.

Mounier, E. (2001). Le Personalisme. Paris: PUF.

Nové-Josserand, F. (2009). L'étonnante histoire des Maisons familiales rurales. Paris: L'Harmattan.

Puig-Calvó, P. (2006). Los centros de formación por alternancia: desarrollo de las personas y de su medio. Consulté le julio 2019, sur https://www.tdx.cat/handle/10803/9345?sh ow=full: $\quad$ http://www.tdx.cat/TDX0129109-133410

Puig-Calvó, P. (2003). Les centres familiaux de formation par alternance: développement des personnes et des milieux. Tours: Universoté de Tours (Memoire DURF).

Puig-Calvó, P. (2019). Formation des Formateurs PROCEFFA. Yaoundé (Cameroun): AIMFR. 
Puig-Calvó, P. (2019). Formation integrale. YAOUNDÉ: PROCEFFA.

Rogers, C. (2013). Liberté pour apprendre. Paris: Dunod.

Rosa, H. (2018). Resonance.Une socilogie de la realtion au Monde. Paris: La découverte.

\section{Rosnay, A. D. (1975). Le macroscope vers une vision globale. Paris: Seuil.}

Sanselme, F. (2000). Les Maisons Familiales Rurales. L'ordre symbolique d'une institution scolaire. Rennes: Presse Universitaires de Rennes.

Vanhulle, S., Merhan, F., \& Ronveaux, C. (2007). Alternances en formation. Bruxelles: de Boeck.

\footnotetext{
${ }^{\mathrm{i}}$ Dans le présent article, nous utiliserons le terme générique CEFFA (Centre Éducatif Familial de Formation par Alternance) pour présenter ces expériences qui se déroulent dans les centres de formation professionnelle par alternance du type EFA (École Familiale Agricole), EFR (École Familiale Rurale), MFR (Maison Familiale Rurale), IFER (Institut de Formation en Entrepreneuriat Rural) ou FFS (Family Farm School).
}

${ }^{\text {ii }}$ La qualification renvoie ici à l'acquisition des compétences (savoirs, savoir-faire et savoir-être) nécessaires à l'exercice d'un emploi ou d'un métier.

iii La formation des producteurs en activité se déroule souvent sous forme de formation modulaire.

iv La Fédération des Écoles et Maisons Familiales Rurales du Cameroun (FEMAFARC), n'ayant pas adhéré à l'ACEFFA, fonctionnait de manière autonome. En outre, la fusion entre la CNEFAC et l'ACEFFA n'ayant pas été possible, cette dernière a dû mettre fin à ses activités.

${ }^{\mathrm{v} D i x}$ (10) EFA furent créées à cette époque parmi lesquels l'EFA de MANDJOU, l'EFA de KOUMEBONIS, l'EFA de GAROUA-BOULAI, l'EFA de BORONGO, l'EFA de MBITOM, l'EFA de KAIGAMA, l'EFA de MARARABA, l'EFA de MAMBAYA, I'FA d'ESSANDJANE, l'EFA de BELABO. Ces EFA étaient accompagnées par la
Coordination Régionale des Écoles Familiales Agricoles de l'Est Cameroun (CREFAEC) fondée par l'Abbé André KRYNSKI, alors Vicaire Épiscopal de l'Archidiocèse de Bertoua. Celle-ci bénéficiait à son tour de l'appui technique de la CNEFAC dans la promotion des EFA et la formation des formateurs.

vi Le philosophe français Emmanuel Mounier (1905-1950) est le fondateur du personnalisme. Pour ce courant de pensée, la valeur principale est le respect de la personne. Ainsi, une action est bonne si elle respecte la personne et contribue à son épanouissement.

vii André Duffaure (1924-1997), ingénieur agronome, fut responsable pédagogique de l'Union Nationale des Maisons Familiales Rurales de France de 1948 à 1956, puis Directeur de 1957 à 1990. Considéré comme l'un des pionniers de l'alternance des CEFFA, il reçoit en 1956le Prix national de pédagogie pour ses travaux sur l'alternance.

viii Alors que l'individualisme est caractérisé par un mouvement de repli sur soi, du sujet lui-même, le personnalisme Emmanuel Mounier se caractérise par une ouverture aux autres. Emmanuel Mounier recommande à cet effet que «nous devons nous libérer de l'individu pour nous ».

ix Le pédagogue français Roger Cousinet (18811973), dont la pédagogie est centrée sur l'activité de l'apprenant, recommandait qu'il n'y ait pas disjonction entre l'activité de l'apprenant et son milieu de vie.

${ }^{\mathrm{x}}$ Le pédagogue américain John Dewey (1859-1952) recommandait qu'on relie les activités d'apprentissage à l'école aux expériences de la vie familiale, sociale de l'apprenant. Sa célèbre formule «learning by doing » (apprendre en faisant) montre que c'est par l'expérience que l'on apprend.

${ }^{\mathrm{xi}}$ Le psychopédagogue américain Carl Rogers (1902-1987) aura enrichi la pensée sur l'alternance par son approche humaniste, centrée sur la personne. Dans son ouvrage Liberté pour apprendre?, il pense que le savoir qui influence profondément le comportement de la personne est celui qu'elle a découvert et s'est approprié luimême.

xii La SAFACAM (Société Africaine Forestière et Agricole du Cameroun), qui fait dans l'hévéaculture et la palmeraie, est promotrice de l'EFA de Dizangué ; tandis que la PHP (Société des Plantations du Haut-Penja), qui cultive et exporte la banane, a créé l'EFA de Njombé PHP. 
Birwe, B., \& Puig-Calvó, P. (2019). Les Centres Éducatifs Familiaux de Formation par Alternance en Afrique: le cas du Cameroun...

\section{Informations sur l'article / Article Information}

Envoyé le: $31 / 07 / 2019$

Approuvé le: 17/10/2019

Posté le: 19/12/2019

Received on July 31th, 2019

Accepted on October 17th, 2019

Published on December, 19th, 2019

Contributions dans l'article: Les auteurs étaient responsables de toutes les étapes et des résultats de la recherche, à savoir: l'élaboration, l'analyse et l'interprétation des données; rédiger et réviser le contenu du manuscrit et; approbation de la version finale publiée.

Author Contributions: The author were responsible for the designing, delineating, analyzing and interpreting the data, production of the manuscript, critical revision of the content and approval of the final version published.

Conflits d'intérêts: Les auteurs ont déclaré qu'il n'y avait pas de conflit d'intérêts concernant cet article.

Conflict of Interest: None reported.

\section{Orcid}

Benoit Birwe

(iD) http://orcid.org/0000-0003-0169-4633

Pedro Puig-Calvó

(iD) http://orcid.org/0000-0002-1367-6478

\section{Comment citer cet article / How to cite this article}

APA

Birwe, B., \& Puig-Calvó, P. (2019). Les Centres Éducatifs Familiaux de Formation par Alternance en Afrique: le cas du Cameroun. Rev. Bras. Educ. Camp., 4, e7325. DOI: http://dx.doi.org/10.20873/uft.rbec.e7325

ABNT

BIRWE, B. ; PUIG-CALVO, P. Les Centres Éducatifs

Familiaux de Formation par Alternance en Afrique: le cas du Cameroun. Rev. Bras. Educ. Camp., Tocantinópolis, v. 4, e7325, 2019. DOI:

http://dx.doi.org/10.20873/uft.rbec.e7325 